\title{
Simple laminar dynamos: from two rolls to one
}

\author{
DAVID MOSS* \\ School of Mathematics, University of Manchester, Oxford Rd \\ Manchester M13 9PLUK
}

(Received 26 June 2007; in final form 19 September 2007)

\begin{abstract}
The study of laminar flows with simple streamlines in conducting fluids that might support kinematic dynamo action has a long history. Early successful examples include the Gailitis (1970) dynamo, which has two meridional rolls. We show here that a single meridional roll, considered as a limit of a modified Gailitis-like dynamo, can also support kinematic dynamo action in a deep spherical shell. This flow has a claim to be the simplest such flow possible.
\end{abstract}

Keywords: Magnetic fields; MHD; Laminar flow dynamos; Dynamo theory

\section{Introduction}

Gailitis (1970; see also Moffatt 1978) showed that a simple axisymmetric laminar flow, consisting of a meridional roll in each of the northern and southern hemispheres of a sphere of conducting fluid, can drive dynamo action resulting in the generation of a steady non-axisymmetric magnetic field. His analysis was restricted to the limit in which the minor radii of the rolls was small compared to the other dimensions of the system. Moss (2006; hereafter M2006), in a numerical study, showed that Gailitis' results remain approximately valid for rolls with minor radii of the order of the radius of the embedding sphere (see also Gailitis 1993, 1995). M2006 also showed that if the sense of flow in one of the rolls was reversed, then a new class of unsteady solutions could be found.

Dudley and James (1989; hereafter DJ) gave a comprehensive survey of laminar flows that could drive dynamo action in a conducting sphere. [Gubbins et al. (2000a, 2000b) made further extensive surveys of dynamo action by steady flows in spheres.] One of DJ's simplest examples was a meridional flow in a single roll passing through both hemispheres (radial velocity $\propto \cos \theta, \theta$ the polar angle) together with a simple latitudeindependent differential rotation (absent in the Gailitis-like examples of M2006). This flow supported oscillatory dynamo action with a non-axisymmetric magnetic field, for sufficiently rapid motion.

\footnotetext{
*Email: moss@ma.man.ac.uk
} 
The question then arises: what is the simplest laminar flow that can support dynamo action in a spherical shell? We show that flow in a single meridional roll can support dynamo action in the absence of differential rotation, and demonstrate the relation between this dynamo and those driven by the "reversed Gailitis"-type flows, studied by M2006. Is there a connection between the simple laminar dynamo of DJ and the Gailitis dynamo? We investigate these and other issues.

\section{Motivation}

Figure 1(a) shows the streamlines of a reversed "finite Gailitis-like" flow of the kind shown by M2006 to support an oscillatory kinematic dynamo, and figure 1(c) shows the meridional part of the DJ flow. Now let the rolls in figure 1(a) approach more closely to the equator, as in figure 1(b). The flow is oppositely directed immediately above and below the equator, and in the limit the effective flow in this region will be small, and the net flow will approach a single roll of the general form considered by DJ as shown in figure 1(c).

Remember, however that in the DJ flow, a differential rotation is also present. Thus this investigation proceeds via the following steps. (1) Show that a "single roll" flow without differential rotation can drive dynamo action; (2) show that as the "reversed Gailitis" rolls become larger and move nearer to the equator, then there is a smooth transition to the result (1); (3) reproduce the DJ result; (4) investigate dynamo action with the meridional part of the DJ flow, but no differential rotation. We also make a

(a)

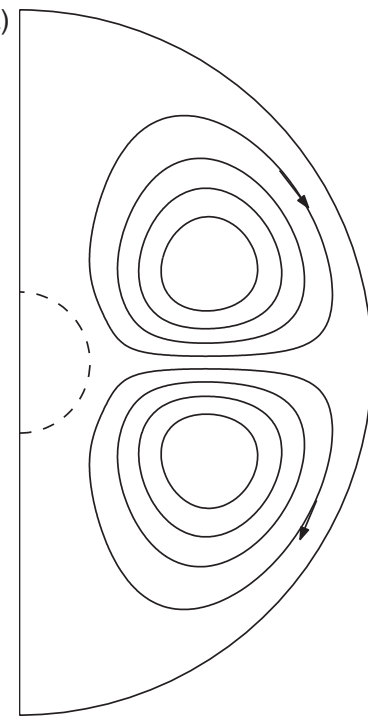

(b)

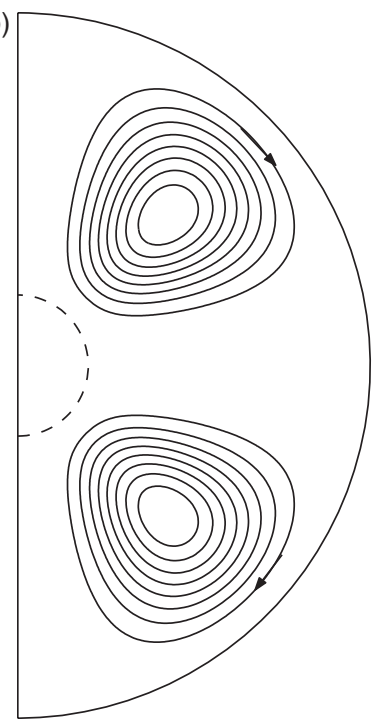

(c)

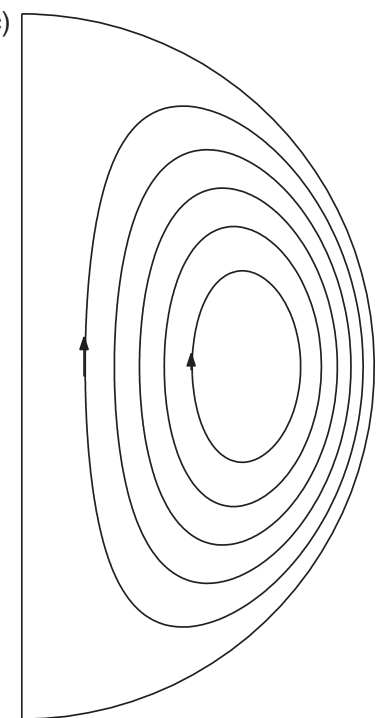

Figure 1. Cross-sections in a meridian plane showing typical streamlines of a reversed Gailitis-like flow for (a) $m=4, n=4$, flow at high latitudes and (b) $m=4, n=1$ with $A \rightarrow-A$ in the southern hemisphere, flow extending to near the equatorial plane. (c) Shows the streamlines of the meridional part of Dudley and James' (1989) flow. In (a) and (b), the dashed contour shows the inner boundary of the flow region. In (c), the flow extends to the centre. 
further step (5), to show that an asymmetrically located single roll can also drive dynamo action.

\section{The numerical code}

We integrate the standard MHD equation

$$
\frac{\partial \mathbf{B}}{\partial \tau}=\nabla \times(\mathbf{v} \times \mathbf{B}-\nabla \times \mathbf{B}) .
$$

using the code and procedure described in M2006. (The diffusivity $\eta$ has been assumed to be uniform, and so does not appear explicitly in this dimensionless form of the induction equation. The length is scaled with $R *$, the radius of the fluid sphere, and the unit of time is $R_{*}^{2} / \eta$.) Briefly, the code expresses the magnetic field in terms of "superpotentials" $\Phi$ and $\Psi$ for the poloidal and toroidal parts of the field, $\mathbf{B}=\mathbf{B}_{P}+\mathbf{B}_{T}=$ break $\nabla \times(\Phi \hat{\mathbf{r}})+\nabla \times \nabla \times(\Psi \hat{\mathbf{r}})$ (e.g. Chandrasekhar 1961), and uses a modal expansion in azimuth. In meridional planes, second-order spatial derivatives are used on a finite difference grid with $M$ points distributed uniformly in fractional radius $r_{0} \leq r \leq 1$, and $N$ points uniformly over polar angle $\theta, 0 \leq \theta \leq \pi$. Equation (1) is timestepped using a semi-implicit Dufort-Frankel method of second-order accuracy in time.

The code is used in linear form, only $k=1$ solutions (azimuthal dependence $e^{\mathrm{i} k \phi}$ ) are considered, the field at $r=1$ is fitted to a vacuum exterior field, and an arbitrary zero boundary condition $\Phi=\Psi=0$ is applied at the inner boundary at radius $r_{0}$. We take $r_{0}=0.2$ for the majority of the computations discussed in Sections 4 and 6. In M2006, it was shown that using overshoot-type boundary conditions, simulating $\Phi$ and $\Psi$ going to zero at distance $\delta$ below the inner boundary by writing $\partial(\Phi, \Psi) / \partial r=(\Phi, \Psi) / \delta$, did not affect solutions significantly. In many cases $M=61, N=101$ is found to be adequate. For some cases, and also to check convergence, a higher resolution, up to $M=N=201$, is used. (Although computations with $M, N>201$ could in principle be handled quite readily, this code matches the poloidal field at the outer boundary to a potential field by a modification of the matrix multiplication method of Jepps (1975), and computation of this matrix by standard library routines becomes difficult for larger values of $\mathrm{N}$. Thus, it was decided to restrict computations to $N \leq 201$, with subsequent implicit restriction on attainable magnetic Reynolds numbers.)

The meridional flow is defined by a time-independent streamfunction $\psi$, so that

$$
\mathbf{v}=\left(\frac{1}{r^{2} \sin \theta} \frac{\partial \psi}{\partial \theta},-\frac{1}{r \sin \theta} \frac{\partial \psi}{\partial r}, v_{\phi}\right),
$$

with respect to a spherical polar coordinate system $(r, \theta, \phi)$. We assume throughout that the density is uniform, and so does not explicitly appear in (2). Gross symmetry properties of both magnetic and velocity fields are described by a parity parameter $P$, where $P=+1 /-1$ for fields with radial components that are respectively of even/odd symmetry with respect to the equator (e.g. Moss et al. 1991). It is convenient to use a more general definition of the parity of magnetic and velocity fields by defining

$$
P=\frac{E^{+}-E^{-}}{E^{+}+E^{-}}
$$


where $E^{+}, E^{-}$are the energies in the part of the relevant field that are respectively symmetric, antisymmetric with respect to the equatorial plane (see, e.g., Moss et al. 1991). Equation (1) was integrated until an eigenmode was reached, showing an exponential growth of the global magnetic energy with time, with unchanging spatial structure (with appropriate allowance for cases of oscillatory growth).

\section{The reversed Gailitis-type flows}

\subsection{Two roll and one roll flows}

The meridional velocity is now given in dimensionless form by a streamfunction $\psi$, where

$$
\psi=A\left(r-r_{0}\right)^{2}(1-r)^{2} \sin ^{m} \theta \cos ^{n} \theta .
$$

Here $m$ and $n$ are positive integers. $A$ is an adjustable constant that controls the magnetic Reynolds number of the flow for given $m$ and $n$; we define $R m=V R_{*} / \eta$, where $V$ is the r.m.s. velocity of the flow and $R *$ and $\eta$ are the radius and resistivity of the fluid sphere (this definition of $R m$ is slightly different from that of M2006). Thus when $n$ is even and $A>0$, motions are upwards near the north pole and downwards near the south pole, whereas if $n$ odd, flow is upwards near both poles. We can thus construct a "reversed Gailitis"-type flow either by taking $n$ to be even, or by allowing $n$ odd and reversing the sign of $A$ in the southern hemisphere - this will be the case where $n=1$ below. Larger values of $m, n$ give more localised velocity distributions. To some extent the value of $m$ controls the angular distance of the stagnation points of the rolls from the axes $\theta=0, \pi$, and $n$ controls the angular distance from the equatorial plane $\theta=\pi / 2$ - see, e.g. figure 1. All these flows are antisymmetric with respect to the equatorial plane, with parity -1 . We present in figure 2 the values of the marginal magnetic Reynolds number, $R m_{\mathrm{c}}$, as $n$ varies for $m=4$. In each case with $n>0$, the dynamo is oscillatory at marginal excitation. The "one-roll" case $m=4, n=0$ [figure 3(a)] was also investigated. Dynamo action again occurs, with steady fields near marginal excitation, with parity $P \approx-0.90$.

These calculations were all initially at a spatial resolution $M=61, N=101$. These results were confirmed by some additional calculations performed with $M=N=201$. For example, the single roll flows with $m=4, n=0$ have marginal value $R m_{c} \approx 350$ at $M=61, N=101$, whereas at the higher resolution $R m_{c} \approx 335$. We thus deduce that the results are reliable. For the $n>0$ flows, the results of M2006 also show that $M=61$, $N=101$ is adequate. Very limited experimentation suggests that the one roll solutions are quite robust with respect to the depth of the shell, for example, steady dynamo solutions were readily found with $r_{0}=0.7$. Then the marginal Reynolds number is $R m_{c} \approx 990$. Note that the two marginal values determined scale approximately with the inverse of the shell thickness.

\subsection{The transition from two roll to one roll solutions}

In figure 2, values of $R m_{c}$ are plotted against $n$, where the single roll solution of Section 4.1 corresponds to $n=0$. It can be seen that the points lie approximately on a 


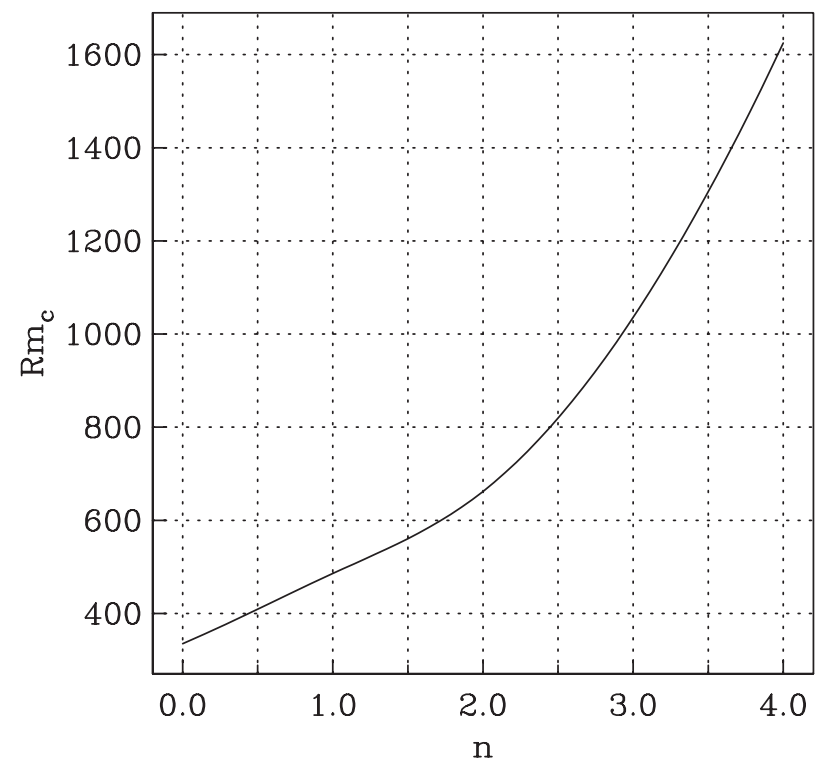

Figure 2. Marginal value of $R m$ against $n$ for flows with $m=4$.

smooth curve. Also note that the two-roll solutions are oscillatory at marginal excitation, whilst the one-roll solution with $n=0$ is steady.

\subsection{Transition to steady growth}

In Section 4.2, we noted that although there was a smooth transition from two-roll solutions with $n>0$ to the single roll solution with $n=0$, the $n>0$ solutions were oscillatory near marginal excitation, whereas the $n=0$ was steady. Thus, for the case $n=1$ we also investigated the nature of the solutions as $R m$ increases. In figure 4 , we plot the growth rate $\Gamma$ of the field against $R m$ for $m=4, n=1$. There is a bifurcation from oscillatory to steady growth at $2.1 \times 10^{3} \mathrm{Rm} 2.3 \times 10^{3}$.

\section{Dudley and James flow}

DJ's flow is given by

$$
\begin{aligned}
\psi & =\epsilon R \sin (\pi r) \sin ^{2} \theta, \\
v_{\phi} & =R \sin (\pi r) \sin \theta,
\end{aligned}
$$

with $\epsilon=0.17$. Streamlines of the meridional part of this flow are shown in figure 1(c); the flow parity again is -1 .

Using the lower spatial resolution $M=61, N=101$ and taking $r_{0}=0.01$ we determined the marginal Reynolds number for the flow (5) as $R_{c} \approx 158$, with frequency $\omega \approx 34$, to be compared with the DJ values $R_{c} \approx 155, \omega \approx 34$. This agreement appears satisfactory.

We then set $v_{\phi}=0, \epsilon=1$ and, with resolution $M=61, N=101$, found dynamo action when $R R_{c} \approx 860$. The fields are steady with parity $P \approx-0.92$ when $R \approx R_{c}$. 
However, when the spatial resolution is increased, there is no evidence of convergence; at $M=N=201$ growing fields were not found at accessible values of $R$. Thus, it cannot be deduced that this flow can support dynamo action.

At first sight, this negative, or at least inconclusive, result seems hard to reconcile with the results of Section 4.1 for the one-roll case with $n=0$. However, there are significant differences between the meridional part of the DJ flow and the $m=4, n=0$ flow of Section 4.1. In the latter case $u_{r}, u_{\theta} \rightarrow 0$ at all boundaries of the computational region, whereas the DJ flow has $u_{r} \neq 0$ at the poles $\theta=0, \pi$, and $u_{\theta} \neq 0$ at the surface. Also, the $m=4, n=0$ flow is significantly more concentrated to the neighbourhood of the stagnation point than the DJ flow (5). In particular, for the larger values of $R$ that appear to be needed for dynamo action in the absence of differential rotation, the nonzero $u_{\theta}$ at the surface advects the magnetic field strongly towards the south pole, decreasing the length scales, increasing dissipation and inhibiting dynamo action. Similar arguments are presented by Gubbins et al. (2000a, 2000b).

One further comment is required. If the results for the DJ flow (5) are to be compared with the single roll solutions of Section 4, then some care must be taken. The DJ definition of the controlling parameter $R$ and the parameter $R m$ used through most of the above are related by

$$
R=\frac{1}{\epsilon} \frac{u_{\mathrm{rms}}}{\left|u_{\mathrm{max}}\right|} R m
$$

\section{A dynamo with a single asymmetric roll}

The question now arises, how much does the excitation of the single roll dynamo, such as that of Section 4.1, depend on the positioning of the roll, in particular on its minor axis lying in the equatorial plane? In order to make a preliminary exploration of this issue, the code was run with velocity field given by $m=6, n=3$ in the northern hemisphere only, i.e. $\mathbf{v}=0$ in the south. Streamlines are shown in figure 3(b). Taking $R m>0$, a steady dynamo was again found to be excited, with marginal $R m$ about 460, and parity $P \approx+0.34$. Increasing the resolution to $M=N=201$ changes the marginal value of $R m$ to about 450 , so the solution appears to be reliable.

With negative values of $R m$, a satisfactory demonstration of dynamo action could not be made for this flow field. It is unclear whether this is a limitation of the spatial resolution used (i.e. the marginal magnetic Reynolds number is too large for $M=N=201$ to give satisfactory spatial resolution), or a more fundamental property of this flow. Clearly, on grounds of continuity, a single roll with $R m<0$ should be capable of driving dynamo action for some positions of the stagnation point/line in the northern hemisphere since, for the single roll of Section 4.1 with stagnation line in the equator, excitation conditions will be identical for flows with magnetic Reynolds numbers $\pm R m$. [Since, given the absence of rotation, if figure 3(a) is inverted in the equatorial plane, a flow with the same $R m c$ will be obtained, but with the velocity now "down" near the axis, rather than "up". In other words, the figure viewed standing on one's head is equivalent to that with negative $R m$.] As the stagnation point moves further from the equator (in the above model, $n$ increases) asymmetry between the flows with positive and negative $R m$ increases, and it appears that excitation with $R m<0$ becomes more difficult. The case computed above is rather extreme - a milder 
(a)

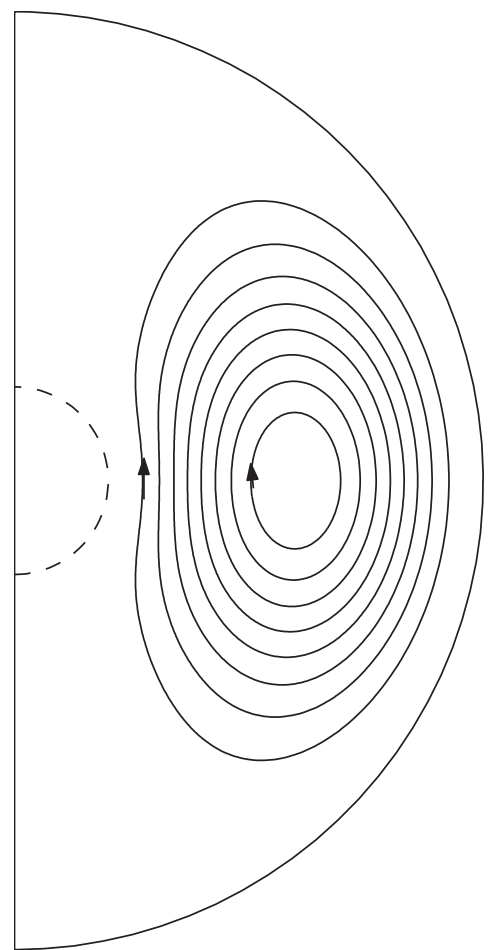

(b)

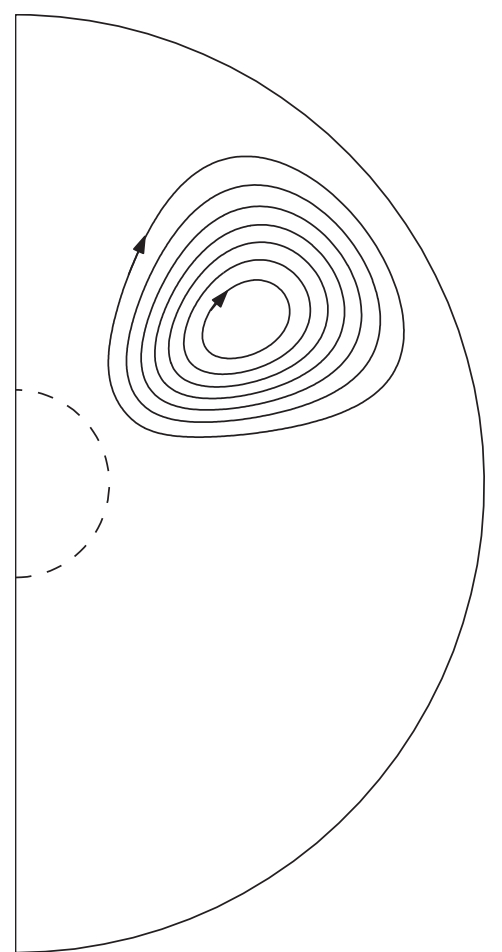

Figure 3. Cross-sections in a meridian plane showing streamlines of single roll flows with (a) $m=4, n=0$, and (b) $m=6, n=3$ and flow in the northern hemisphere only.

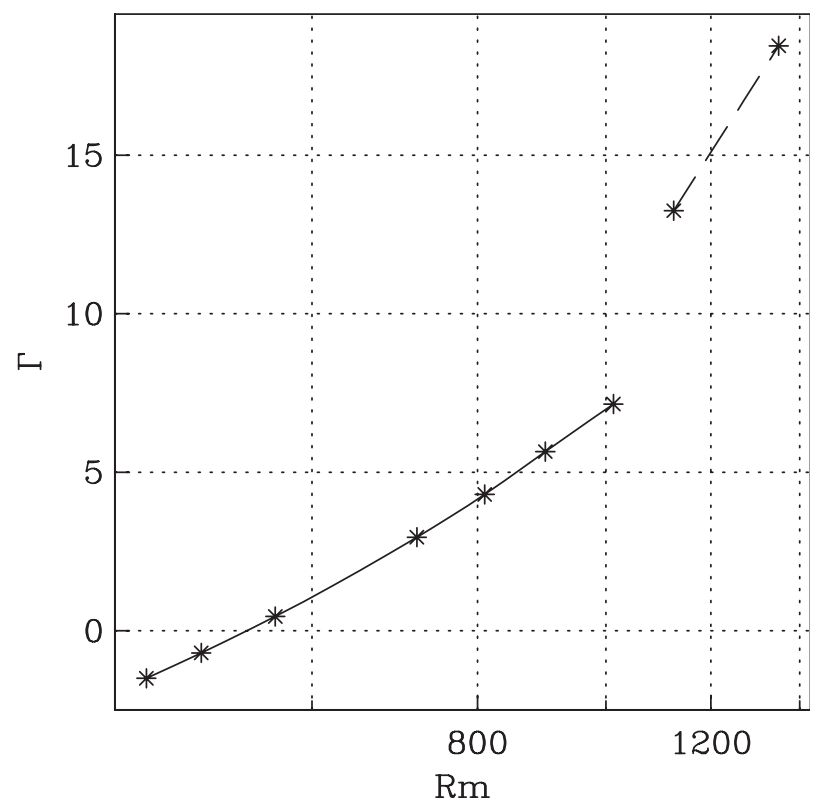

Figure 4. Bifurcation diagram with $R m$ increasing, for case $m=4, n=1$ with change of sign in $A$ (4) in the southern hemisphere. The broken curve links steady solutions, the remainder are oscillatory. 
asymmetry with a displaced stagnation point but the flow still extending through both hemispheres could be envisaged - but this issue was not pursued.

\section{Conclusions}

It has been demonstrated numerically that a single (axisymmetric) meridional roll can drive kinematic dynamo action in a deep spherical shell of conducting fluid. The magnetic fields generated are necessarily nonaxisymmetric (as implied by a variety of anti-dynamo theorems). This flow seems to be simpler than that supporting any previously investigated dynamo in such a geometry, where the fluid motions are confined to a finite region. [The Ponomarenko dynamo, Ponomarenko (1973), is another very simple example, but there the fluid motions extend to infinity.] There is freedom to locate the roll asymmetrically with respect to the equatorial plane, even to the extent that velocities are non-zero in only one hemisphere.

Further, when the roll is symmetrically positioned with respect to the equatorial plane, the magnetic fields generated can, near marginal values of the flow velocity, be considered to be a limiting case of dynamos with two rolls, oppositely directed in the northern and southern hemispheres, in what might be called a "reversed finite Gailitis" configuration. It is difficult to envisage a less complex flow that might support dynamo action in a sphere or spherical shell.

"One roll" dynamos are of interest in the context of dynamo theory but astrophysical situations with such symmetry breaking flows are however hard to envisage. Large scale flows in astrophysical bodies are usually driven by rotation or large-scale axially symmetric magnetic fields (e.g. Mestel 1965), and consist of sets of cellular flows symmetric with respect to the equatorial plane with flow parity +1 . Large scale stellar magnetic fields with symmetry axes perpendicular to the rotation axis can, in principle, drive flows with equatorial asymmetry, but then the flows cannot be used to generate the fields! Speculatively, perhaps flows in dynamo experiments where the fluid motions are driven by propellers at one end of a cavity, might contain a component of similar geometry.

The simplicity of these nonaxisymmetric dynamos driven by simple meridional flows, compared with the care necessary to choose velocity fields to generate axisymmetric magnetic fields, is striking. Of course, the examples discussed here all ignore back reactions of the Lorentz force onto the flow; in a more self-consistent dynamical treatment the meridional flow would be modified and a differential rotation would arise.

\section{Acknowledgements}

The author is grateful to Dr J.-F. Pinton for bringing the papers of Gailitis (1993, 1995) to his attention. Leon Mestel is thanked for comments on a preliminary version of the text. Constructive comments by the referees (D. Gubbins and F. Plunian) improved the presentation. 


\section{References}

Chandrasekhar, S., Hydrodynamic and Hydromagnetic Stability, 1961 (Clarendon Press: Oxford).

Dudley M.L., James R.W. Time-dependent kinematic dynamos with stationary flows. Proc. R Soc. Lond. A. 1989, 425, 407-429.

Gailitis A. Self-excitation of a magnetic field by a pair of annular vortices. Mag. Gidrod, 1970, 6, 19-22 (English translation: Magnetohydrodynamics, 6:14-17).

Gailitis A. Magnetic field generation by axisymmetric flows of conducting liq-uids in a spherical stationary conductor cavity. Mag. Gidrod, 1993, 29, 3-14 (English translation: Magnetohydrodynamics, 29:107-115).

Gailitis A. Magnetic field generation by the axisymmetric conducting fluid flow in a spherical cavity of a stationary conductor. 2. Mag. Gidrod. 1995, 31, 39-43 (English translation: Magnetohydrodynamics, $31: 38-42)$.

Gubbins, D., Barber, C.N., Gibbons, S. and Love, J.J., Kinematic dynamo action in a sphere. I Effects of differential rotation and meridional circulation on solutions with axial dipole symmetry. Proc. $R$ Soc. Lond. A, 2000a, 456, 1333-1353.

Gubbins, D., Barber, C.N., Gibbons, S. and Love, J.J., Kinematic dynamo action in a sphere. II Symmetry selection. Proc. R. Soc. Lond. A, 2000b, 456, 1669-1683.

Jepps, S.A., Numerical models of hydromagnetic dynamos. J. Fluid Mecha., 1975, 67, 625-646.

Mestel L. In: Kuiper G, editor. Meridian Circulation in Stars. In Stars and stellar systems, Vol. 8, 1965 (Chicago, IL: University of Chicago Press), pp. 297-326.

Moffatt, H.K., Magnetic field generation in electrically conducting fluids, 1978 (CUP: Cambridge).

Moss, D., Numerical simulation of the Gailitis dynamo. GAFD, 2006, 100, 49-58.

Moss, D., Tuominen, I. and Brandenburg, A., Nonlinear nonaxisymmetric dynamo models for cool stars. Astron. Astrophys., 1991, 245, 129-135.

Ponomarenko Yu. B. Theory of the hydromagnetic generator. Zh Prikl Melch Telch Fiz (USSR), 1973, 6, 47-51 (English translation: J. Appl. Mech. Techn. Phys. 14:775-778). 